\title{
Comparison of different biopsy forceps models for tissue sampling in eosinophilic esophagitis
}

\author{
Christian Bussmann ${ }^{1,}$ *, Alain M. Schoepfer ${ }^{2,}{ }^{*}$, Ekaterina Safroneeva ${ }^{3}$, Nadine Haas ${ }^{3}$, Sébastien Godat ${ }^{1}$, \\ Christine Sempoux ${ }^{4}$, Hans-Uwe Simon ${ }^{5}$, Alex Straumann ${ }^{6}$ \\ Institutions are listed at end of article.
}

Corresponding author Alain Schoepfer, MD

Division of Gastroenterology and Hepatology

Centre Hospitalier Universitaire Vaudois (CHUV)

Rue du Bugnon 44/07/2409

$\mathrm{CH}-1011$, Lausanne Switzerland

Fax: +41-21-3144718

alain.schoepfer@chuv.ch
Background and aims: Eosinophilic esophagitis (EoE) is a mixed inflammatory and fibrostenotic disease. Unlike superficial inflammatory changes, subepithelial fibrosis is not routinely sampled in esophageal biopsies. This study aimed to evaluate the efficacy and safety of deep esophageal sampling with four different types of biopsy forceps. Patients and methods: In this cross-sectional study, esophageal biopsies were taken in 30 adult patients by one expert endoscopist. Biopsies sampled from distal esophagus using a static jaw forceps (Olympus, FB-11K-1) were compared with proximal biopsies sampled with static jaw (Olympus, FB-45Q-1), alligator jaw (Olympus, FB-210K), and large-capacity forceps (Boston Scientific, Radial Jaw 4). One pathologist calculated the surface area of epithelial and subepithelial layers in hematoxylin and eosin (H\&E)-stained biopsies.

Results: Subepithelial tissue was acquired in 97\%

\section{Introduction}

$\nabla$

Eosinophilic esophagitis (EoE) is a chronic, inflammatory disorder of the esophagus, rapidly increasing in incidence, and characterized clinically by symptoms related to esophageal dysfunction and histologically by an eosinophil-predominant inflammation [1-3]. In itself, this definition underscores the importance of the histopathological assessment of the esophageal tissue.

Systematic evaluation of scarce surgical resection specimens as well as findings from endoscopic ultrasound studies have convincingly shown that, in EoE, the inflammatory processes are not restricted to the squamous epithelium of the esophagus but also take place in the deeper esophageal wall layers, in particular the lamina propria of the mucosal layer, the submucosal layer, and even the muscularis and adventitia [4-8]. It is well estab-

* These authors contributed equally. (static jaw FB-11K-1), 93\% (static jaw FB-45Q-1), $80 \%$ (alligator jaw), and 55\% (large-capacity) of samples. Median (interquartile [IQR]) ratios of surface area of epithelial to subepithelial tissue were: static jaw FB-45Q-1, 1.07 (0.65-4.465); static jaw FB-11K-1, 1.184 (0.608-2.545); alligator jaw, 2.353 (1.312-4.465); and large-capacity, 2.71 (1.611-4.858). The static jaw models obtained a larger surface area of subepithelial tissue compared with the alligator jaw $(P<0.001$ and $P=$ 0.037 , for FB-11K-1 and FB-45Q-1, respectively) and the large-capacity forceps $(P<0.001$, for both static jaw models). No esophageal perforations occurred.

Conclusions: The static jaw forceps models allowed sampling of subepithelial tissue in $>90 \%$ of biopsies and appear to be superior to alligator or large-capacity forceps in sampling larger amounts of subepithelial tissue.

lished that uncontrolled inflammation in EoE can lead to subepithelial fibrosis characterized by excess deposition of extracellular matrix proteins $[9,10]$. This so-called esophageal remodeling leads to stricture formation, which increases the risk of food bolus impactions $[11,12]$. Subepithelial fibrosis might contribute to EoE symptoms despite apparent mucosal healing. As such, processes taking place in deeper layers of the esophageal wall most likely contribute to symptom generation in EoE [13].

In EoE, it would be advantageous to get biopsies that encompass subepithelial tissue as well as epithelium. The reasons for this are twofold: (i) it would be of interest to physicians and scientists alike to be able to examine disease-specific alterations occurring more deeply in the esophageal wall and to improve our understanding of the pathophysiology of EoE; and (ii) it would help pathology technicians to properly orient biopsy specimens (epithelial side up), which in turn would make it easier for the pathologist to evaluate the 
distinct EoE-associated features, such as basal cell hyperplasia or papillary elongation [14].

Although subepithelial esophageal tissue is not routinely sampled, several models of biopsy forceps currently on the market can be used to potentially obtain this tissue [15]. However, there are no studies so far that have systematically assessed the success rates for sampling subepithelial tissue achieved by endoscopists using different biopsy forceps models [16]. Furthermore, it is unknown whether sampling of biopsies containing subepithelial tissue is associated with complications, such as bleeding necessitating an endoscopic or surgical intervention or esophageal perforation.

Given the lack of studies that systematically examine the use of different biopsy forceps in patients with EoE, we aimed to evaluate the rates with which four different biopsy forceps models can be used to successfully obtain biopsy specimens containing subepithelial tissue, and the amount of tissue that can be sampled using these forceps. In addition, we also examined whether inclusion of subepithelial tissue is associated with obtaining sections with proper basoapical orientation. Lastly, we examined whether the deeper biopsy sampling is associated with complications, such as bleeding or esophageal perforation.

\section{Patients and methods \\ $\nabla$}

\section{Patients}

In this cross-sectional study, adult EoE patients were recruited (by A.S.) at the Swiss EoE Clinic, Olten, Switzerland. For diagnosis of EoE, patients fulfilled the following published diagnostic criteria: (i) symptoms of esophageal dysfunction; (ii) peak eosinophil count of $\geq 15$ /high-power field (hpf; magnification $\times 400$ ) following an 8-week treatment with proton-pump inhibitors (PPIs) (single standard or double dose); and (iii) exclusion of other disorders that might be associated with esophageal eosinophilia [17]. Patients underwent esophagogastroduodenoscopy (EGD) because they were either symptomatic or else scheduled for a regular follow-up visit. Patients with PPI-responsive esophageal eosinophilia (PPI-REE) were excluded [18].

Patients were recruited from June 2015 to October 2015. Adult patients (18-80 years) who provided written informed consent were included in the study. Patients were excluded if they were pregnant, or receiving anticoagulation treatment that would in- crease the risk for hemorrhage during esophageal biopsy sampling. The study was approved by the local ethics committee (EKNZ 2015/388).

\section{Endoscopy and biopsies}

One EoE expert endoscopist (A.S.) performed the EGDs with biopsy sampling according to a standardized protocol. Four distal and four proximal biopsies were taken from each EoE patient. For the purposes of the study, the distal esophagus was defined as the section spanning the lower half of the esophagus, while the proximal esophagus was defined as the section spanning the upper half of the esophagus. The closed biopsy forceps were passed through the working channel of the endoscope, positioned tangentially to the esophageal wall and then opened. Using suction, the mucosa was pulled towards the tip of the endoscope and then grasped with the biopsy forceps.

The following biopsy forceps models were evaluated: static jaw, FB-11K-1 (Olympus Europa, Hamburg, Germany); static jaw, FB45Q-1 (Olympus Europa); alligator jaw, FB-210K (Olympus Europa), and large-capacity, Radial Jaw 4 (Boston-Scientific, Solothurn, Switzerland,). The characteristics of the different forceps models are presented in $\bullet$ Fig. 1.

Our group recently evaluated the distribution of subepithelial fibrosis in 149 adult EoE patients [19]. No difference was found when fibrosis frequency and severity in the proximal was compared with that in the distal esophagus $(P=0.183)$ [19]. Based on this observation, four biopsy samples were taken in the distal esophagus from all 30 patients, using the static jaw FB-11K-1 forceps. In addition four biopsies were taken in the proximal esophagus in each patient, using one of the other three forceps models. For these proximal esophageal biopsies, the patients were split into three equal groups (10 patients each), and the static jaw FB45Q-1, alligator jaw, and large-capacity forceps were used to take biopsies from the proximal esophagus in the first, second, and third groups, respectively.

\section{Histopathological examination}

All samples were sent to one EoE expert pathologist (C.B.) for processing and evaluation (at the Viollier Institute of Pathology, Allschwil, Switzerland). Sections of thickness $4 \mu \mathrm{m}$ were cut from paraffin blocks and then stained with hematoxylin and eo$\sin (\mathrm{H} \& \mathrm{E})$ for examination by light microscopy. Slides were evaluated using a Zeiss Axio Imager.A2 microscope (Carl Zeiss, Feld-

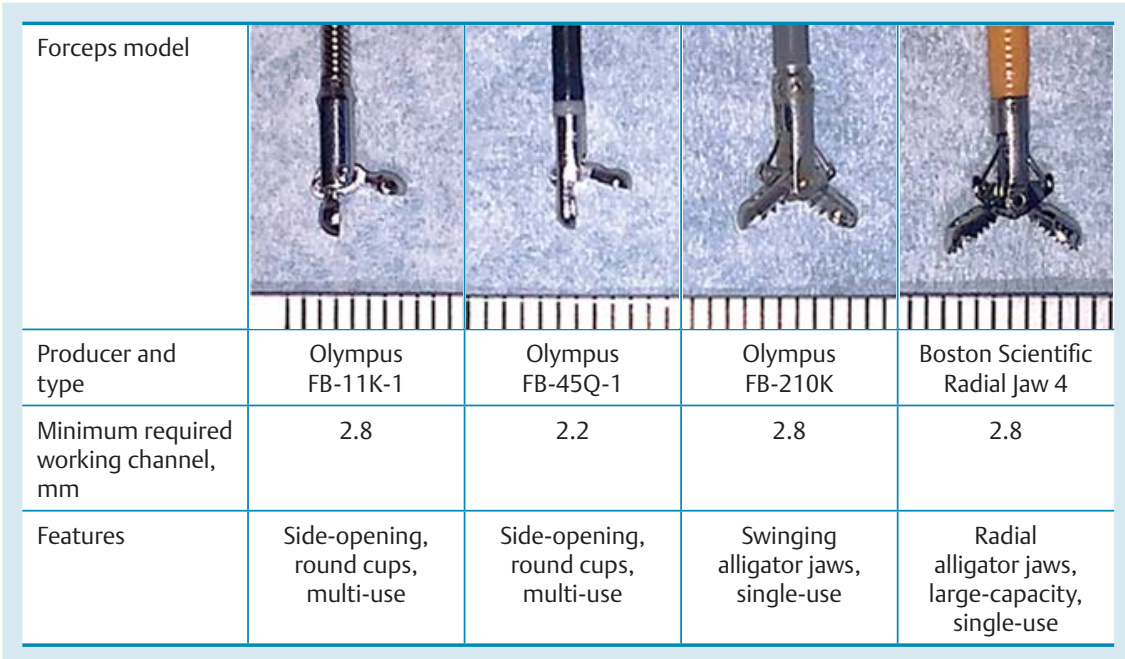

Fig. 1 Characteristics of four models of biopsy forceps used in evaluation of efficacy and safety of deep esophageal sampling. 
bach, Switzerland) with a high power field (hpf) area of 0.260 $\mathrm{mm}^{2}$ (a high power field represents $\times 400$ magnification).

At least 15 interval sections of every esophageal biopsy specimen were surveyed under magnification $\times 100$ (low power field), and the eosinophils in the most densely infiltrated area were counted under high power examination. The following features were recorded: sample orientation (sectioned perpendicularly to the luminal surface, yes vs. no), percentage of the hpf covered by the tissue, peak number of eosinophils/hpf, distribution of eosinophils in a hpf, presence of eosinophilic microabscesses (aggregation of $>10$ eosinophils), basal layer enlargement, and lamina propria fibrosis. Lamina propria fibrosis was evaluated categorically (absent, mild - moderate, severe) following the histopathology protocol of the eosinophilic esophagitis activity index (EEsAI) study [19].

The surface areas of the epithelial and subepithelial layers were calculated by measuring the circumference of these layers ( $\bullet$ Fig. 2). In addition, the ratio of the surface area of the sampled epithelial tissue to that of the subepithelial tissue was calculated for each type of biopsy forceps used.

The assessment of the severity of EoE-associated endoscopic findings, such as white exudates, rings, edema, furrows, and stricture(s), was carried out in accordance with the EoE Endoscopic Reference Score classification and grading system [20].

\section{Statistical analysis}

Data were entered into an Excel spreadsheet (Microsoft Excel 2010; Microsoft Corporation, Redmond, Washington, USA). The statistical analyses were performed using Stata (version 13.1, College Station, Texas, USA). Q-Q plots were used to analyze data distribution. Most data were normally distributed, except for the values of the ratio of the surface area of epithelial tissue to the surface area of subepithelial tissue, which followed a nonparametric distribution. Results of numerical data are presented either as mean (standard deviation [SD]), or as $95 \%$ confidence intervals $(95 \% \mathrm{CI})$ for normally distributed data, or as median (interquartile range $[\mathrm{IQR}])$, for nonparametric data.

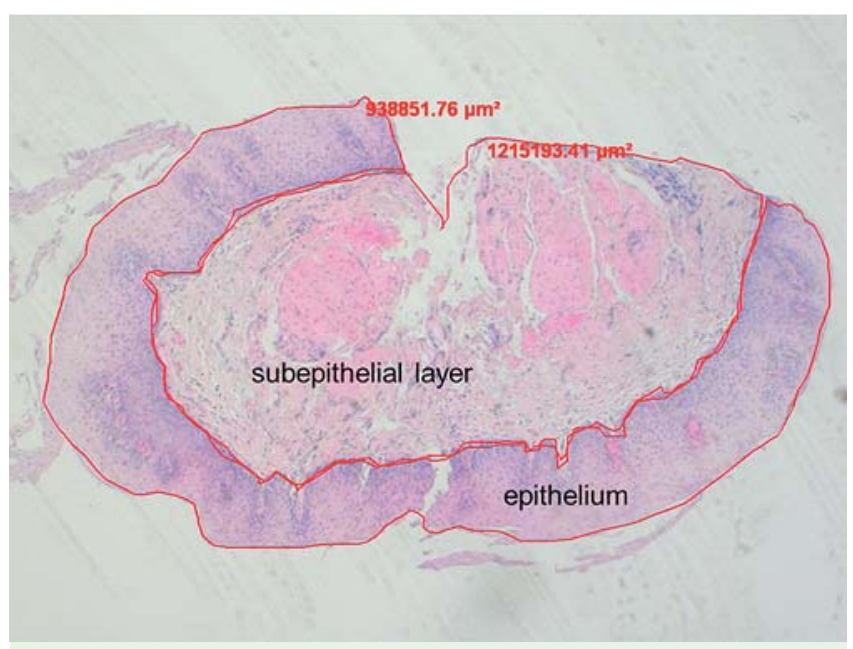

Fig. 2 Biopsy sampling in eosinophilic esophagitis (EoE). The surface area of the epithelial and subepithelial layers was calculated using software and based on measuring the circumference of each individual layer. The section shows a correct basoapical orientation that allows the evaluation of the entire depth of the biopsy. (Biopsy sample acquired with the Olympus FB$11 \mathrm{~K}-1$ forceps.)
To compare two groups with regard to a continuous variable, we used the $t$ test if the variable was normally distributed in both groups and the Wilcoxon rank sum test if the variable had a skewed distribution.

Our study was considered to be an exploratory analysis. However, because of multiple testing and the limited sample size, $P$ values $<0.01$ were considered to be statistically significant.

\section{Results}

\section{$\nabla$}

\section{Clinical, endoscopic, and histologic characteristics} of EoE patients

A total of 30 adult EoE patients from the Swiss EoE Clinic were included (mean [SD] age 45.2 [14.3] years at inclusion; 66.7\% men). The baseline characteristics of these patients are shown in - Table 1. At the time of undergoing EGD, 13 patients (43.3\%) were asymptomatic, while 17 patients (56.7\%) were suffering

Table 1 Clinical, endoscopic and histologic characteristics of patients $(n=30)$ with eosinophilic esophagitis (EoE) and enrolled in the study.

\begin{tabular}{|c|c|}
\hline Age at EoE diagnosis, mean (SD), years & $38.6(17.7)$ \\
\hline Age at inclusion, mean (SD), years & $45.2(14.3)$ \\
\hline Male gender, $\mathrm{n}(\%)$ & $20(66.7)$ \\
\hline Family history of EoE, n (\%) & $2(6.7)$ \\
\hline \multicolumn{2}{|l|}{ Therapies (ever), n (\%) } \\
\hline Oral topical steroids (budesonide or fluticasone) & $28(93.3)$ \\
\hline Elimination diet & $1(3.3)$ \\
\hline Esophageal dilation & $6(20)$ \\
\hline \multicolumn{2}{|l|}{ Therapies at current endoscopy, n (\%) } \\
\hline Oral topical steroids & $23(76.7)$ \\
\hline Elimination diet & 0 \\
\hline Esophageal dilation & $2(6.7)$ \\
\hline No therapy & $5(16.7)$ \\
\hline \multicolumn{2}{|l|}{$\begin{array}{l}\text { EoE symptoms at inclusion (patients may have } \\
\text { reported more than one symptom), } n(\%)\end{array}$} \\
\hline Asymptomatic & $13(43.3)$ \\
\hline Dysphagia & $17(56.7)$ \\
\hline $\begin{array}{l}\text { Food impaction necessitating endoscopic } \\
\text { removal }\end{array}$ & $0(0.0)$ \\
\hline Thoracic pain, not associated with swallowing & $2(6.7)$ \\
\hline Heartburn & $1(3.3)$ \\
\hline Swallowing-associated thoracic pain & $2(6.7)$ \\
\hline Weight loss & $1(3.3)$ \\
\hline Atopic disease, $\mathrm{n}(\%)$ & $25(83.3)$ \\
\hline Allergic rhinoconjunctivitis & $12(40.0)$ \\
\hline Asthma & $8(26.7)$ \\
\hline Allergic rhinoconjunctivitis and asthma & $10(33.3)$ \\
\hline Oral allergy syndrome & $6(20.0)$ \\
\hline Urticaria & $0(0.0)$ \\
\hline Known food allergies & $14(46.7)$ \\
\hline \multicolumn{2}{|l|}{$\begin{array}{l}\text { Endoscopic features (patients may have had } \\
\text { more than one endoscopic feature), } n(\%)\end{array}$} \\
\hline White exudates & $9(30.0)$ \\
\hline Longitudinal furrows & $8(26.7)$ \\
\hline Edema & $18(60.0)$ \\
\hline Corrugated rings & $19(63.3)$ \\
\hline Stricture & $9(30.0)$ \\
\hline \multicolumn{2}{|l|}{ Histologic features (entire esophagus) } \\
\hline $\begin{array}{l}\text { Peak eosinophil count per hpf, median (IQR), } \\
\text { (range) }\end{array}$ & $20(4-35),(0-148)$ \\
\hline Eosinophilic microabscesses, n (\%) & $11(36.7)$ \\
\hline Subepithelial fibrosis, n (\%) & $25(83.3)$ \\
\hline
\end{tabular}

hpf, high power field; IQR, interquartile range. 


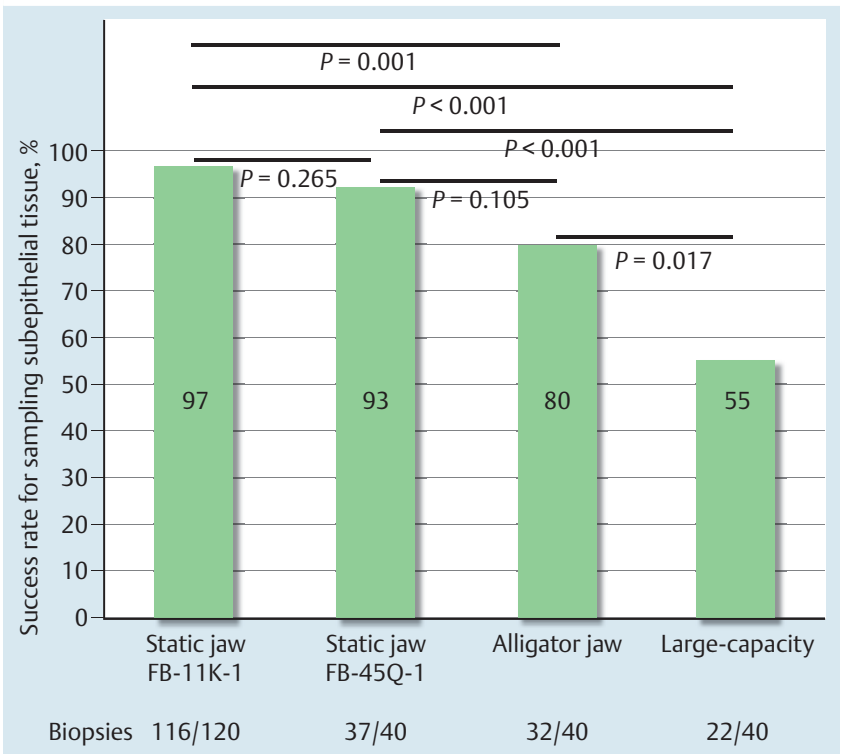

Fig. 3 Success rate for sampling subepithelial tissue. Both static jaw forceps models provided subepithelial tissue in $>90 \%$ of biopsies. The alligator jaw and large-capacity forceps provided subepithelial tissue in $80 \%$ and $55 \%$ of biopsies, respectively.

from dysphagia. The majority of patients (83.3\%) suffered from concomitant allergies.

The endoscopic and histologic characteristics of the EoE patients at the time of endoscopy are shown in $\bullet$ Table 1 . Rings were the most prevalent feature (19/30, 63.3\%), followed by edema (18/30, $60 \%)$, white exudates $(9 / 30,30 \%)$, strictures $(9 / 30,30 \%)$, and furrows $(8 / 30,26.7 \%)$. The median peak eosinophil count was 20/hpf. Eosinophilic microabscesses were found in 11 patients (36.7\%), while subepithelial fibrosis was present in 25 patients (83.3\%).

Acquisition of subepithelial tissue by the four models of biopsy forceps

The success rate for acquisition of subepithelial tissue by the four different biopsy forceps models is shown in $\bullet$ Fig. $\mathbf{3}$.
Subepithelial tissue was acquired in more than $90 \%$ of all biopsies when static jaw type forceps were used: with the static jaw FB$11 \mathrm{~K}-1$, the success rate was $116 / 120$ (97\%, 95\%Cl $94.7 \%-100.3 \%$; with the static jaw FB-45Q-1 it was $37 / 40$ (93\%, 80.5\%-99.7\%). The alligator jaw forceps acquired subepithelial tissue in 32/40 biopsies (80\%, 67.0\%-92.9\%), and the large-capacity forceps in $22 / 40$ ( $55 \%, 38.9 \%-71.1 \%)$.

The use of static jaw and alligator forceps was associated with higher success rates in obtaining subepithelial tissue compared with the large-capacity forceps ( $\boldsymbol{O}$ Fig. $\mathbf{3}$ ). There was no difference between the static jaw FB-11K-1 and static jaw FB-45Q-1 models for success in acquiring subepithelial tissue $(P=0.265)$.

- Fig. 4 shows representative sections as sampled by the four different biopsy forceps models.

Amounts of epithelial and subepithelial tissue acquired The surface areas of the epithelial and subepithelial tissue obtained using the four different biopsy forceps models are shown in - Table2. With regard to the amount of epithelial tissue sampled, the large-capacity forceps provided the largest surface area of epithelial tissue, followed by the alligator jaw, and the two static jaw biopsy forceps (FB-11K-1 and FB-45Q-1). However, when examining the amount of subepithelial tissue acquired, we found that the static jaw FB-11K-1 forceps provided the largest tissue surface area, followed by the static jaw FB-45Q-1, alligator, and large-capacity forceps.

We further evaluated the ratio of surface area of epithelial tissue to that of subepithelial tissue acquired ( $\bullet$ Table 2$)$. A ratio of 1 means that equal amounts of epithelial and subepithelial tissue were acquired. The higher the ratio, the larger is the relative amount of epithelial tissue acquired and the smaller the relative amount of subepithelial tissue. We found that the static jaw FB45Q-1 had the lowest ratio (median 1.07), followed by the static jaw FB-11K-1 (median 1.184), the alligator jaw (median 2.353), and the large-capacity forceps (median 2.71). 0 Table 3 shows the $P$ values for the comparisons between the four different models of biopsy forceps, for surface area of acquired epithelial tissue, for surface area of acquired subepithelial tissue, and for the epithelial/subepithelial surface area ratios.

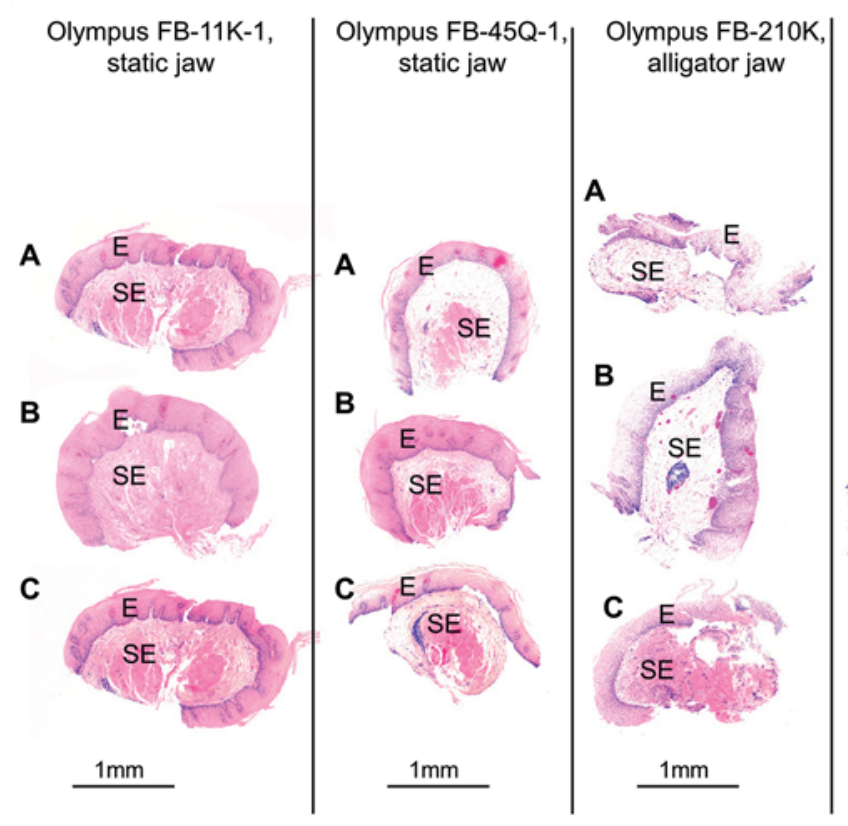

Boston Scientific

Radial Jaw 4, large-capacity forceps

A
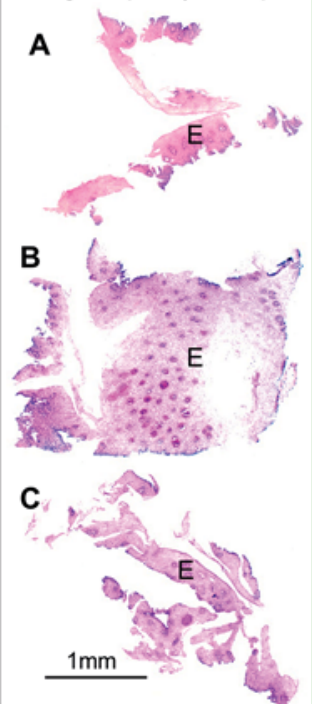

Fig. 4 Representative examples ( $A-C$ ) of sections using the four different models of biopsy forceps. The sections acquired with the large-capacity forceps show a lack of basoapical orientation (observed in $27.5 \%$ of biopsies sampled with this forceps type). E, epithelial layer; SE, subepithelial layer. 


\begin{tabular}{|c|c|c|c|}
\hline & \multicolumn{2}{|c|}{ Surface area, mean (SD), mm² } & \multirow{2}{*}{$\begin{array}{l}\text { Ratio of surface areas epithelial layer/subepithelial layer, } \\
\text { median (IQR) }\end{array}$} \\
\hline & Epithelial tissue & Subepithelial tissue & \\
\hline Static jaw FB-11K-1 & $0.949(0.413)$ & $0.857(0.588)$ & $1.184(0.608-2.454)$ \\
\hline Static jaw FB-45Q-1 & $0.731(0.344)$ & $0.537(0.332)$ & $1.07(0.65-2.327)$ \\
\hline Alligator jaw & $0.957(0.414)$ & $0.372(0.367)$ & $2.353(1.312-4.465)$ \\
\hline Large-capacity & $1.199(0.425)$ & $0.274(0.381)$ & $2.71(1.611-4.858)$ \\
\hline
\end{tabular}

SD, standard deviation; IQR, interquartile range.

Table 3 Pvalues for comparisons, amongst four different biopsy forceps models, for surface area of epithelial tissue acquired, surface area of subepithelial tissue acquired, and ratio of surface area of epithelial tissue to that of subepithelial tissue.

\begin{tabular}{|c|c|c|c|c|c|}
\hline & & $\begin{array}{l}\text { Static jaw } \\
\text { FB-11K-1 }\end{array}$ & $\begin{array}{l}\text { Static jaw } \\
\text { FB-45Q-1 }\end{array}$ & Alligator jaw & Large-capacity \\
\hline \multirow[t]{4}{*}{ Surface area of epithelial tissue } & $\begin{array}{l}\text { Static jaw } \\
\text { FB-11K-1 }\end{array}$ & - & 0.003 & 0.922 & $<0.001$ \\
\hline & $\begin{array}{l}\text { Static jaw } \\
\text { FB-45Q-1 }\end{array}$ & & - & $<0.001$ & $<0.001$ \\
\hline & Alligator jaw & & & - & 0.007 \\
\hline & Large-capacity & & & & - \\
\hline \multirow[t]{4}{*}{ Surface area of subepithelial tissue } & $\begin{array}{l}\text { Static jaw } \\
\text { FB-11K-1 }\end{array}$ & - & 0.001 & $<0.001$ & $<0.001$ \\
\hline & $\begin{array}{l}\text { Static jaw } \\
\text { FB-45Q-1 }\end{array}$ & & - & 0.037 & $<0.001$ \\
\hline & Alligator jaw & & & - & 0.216 \\
\hline & Large-capacity & & & & - \\
\hline \multirow[t]{4}{*}{$\begin{array}{l}\text { Ratio of surface areas, epithelial } \\
\text { tissue/ subepithelial tissue }\end{array}$} & $\begin{array}{l}\text { Static jaw } \\
\text { FB-11K-1 }\end{array}$ & - & 0.560 & 0.144 & 0.004 \\
\hline & $\begin{array}{l}\text { Static jaw } \\
\text { FB-45Q-1 }\end{array}$ & & - & 0.046 & 0.005 \\
\hline & Alligator jaw & & & - & 0.253 \\
\hline & Large-capacity & & & & - \\
\hline
\end{tabular}

\section{Orientation of the biopsy specimens}

We further evaluated whether the presence of subepithelial tissue acquired by the different biopsy forceps models might influence the chance of obtaining properly oriented biopsy specimens (with epithelial side uppermost, sectioned perpendicularly to the luminal surface).

- Fig. 4 shows examples of properly oriented samples, obtained using the static jaw and the alligator jaw models, and badly oriented biopsies obtained using the large-capacity model. Rates for properly oriented esophageal biopsies were: static jaw FB-11K-1 forceps, 112/120 (93.3\%); static jaw FB-45K-1, 34/40 (85\%); alligator jaw, 35/40 (87.5\%); and large-capacity forceps, 29/40 (72.5\%).

When the static jaw FB-11K-1, static jaw FB-45K, and alligator forceps were compared versus the large-capacity forceps, the $P$ values were $<0.001,0.172$, and 0.094 , respectively. No differences regarding the rate of properly oriented biopsies were observed, when the two static jaw and alligator forceps models were compared with one another (static jaw FB-11K-1 vs. static jaw FB45Q-1, $P=0.106$; static jaw FB- $11 \mathrm{~K}-1$ vs. alligator, $P=0.242$; alligator jaw vs. static jaw FB-45Q-1, $P=0.745$ ).

\section{Safety aspects}

When biopsies were acquired using different biopsy models, we observed no case of esophageal perforation or hemorrhage that would necessitate an endoscopic or surgical hemostasis, or blood transfusion, or hospitalization.

\section{Discussion}

In this first study comparing subepithelial tissue biopsy sampling using four different forceps, we have identified the following findings that are relevant to endoscopic and histologic work-up of adult patients with EoE: (i) subepithelial tissue was acquired in more than $90 \%$ of all biopsies using static jaw forceps, in $80 \%$ of all biopsies using alligator jaw forceps, and in only $55 \%$ of biopsies using the large-capacity forceps; (ii) static jaw biopsy forceps models had the lowest ratio of amount of epithelial to subepithelial tissue sampled; (iii) the large-capacity forceps showed the highest percentage (27.5\%) of badly oriented esophageal biopsies; and (iv) deeper biopsy sampling using all biopsy forceps models examined appears to be safe.

So far, most EoE-associated histologic alterations reported in the literature are those observed in the epithelium and they are inflammatory in nature. These features include peak eosinophil count, presence of eosinophilic microabscesses, eosinophil degranulation, papillary elongation, and basal cell hyperplasia of the epithelium [21]. Evidence is increasing that the inflammatory processes in EoE are not restricted to the epithelium but might encompass all esophageal wall layers [4-8]. As yet, our knowledge about pathogenic alterations in the subepithelial layer is limited as this tissue is not routinely sampled. It would be advantageous for several reasons to routinely acquire biopsies that encompass subepithelial tissue as well as epithelium.

First, sampling of subepithelial tissue can improve our understanding of EoE pathogenesis and clarify the relationship be- 
tween symptom severity and biologic activity [13,22]. The pathogenesis of EoE involves inflammatory as well as fibrotic processes. Eosinophilic granulocytes in the subepithelial layer might cause motility disturbances of the adjacent muscle layer [23]. In the long term, untreated eosinophil-predominant inflammation can lead to excessive subepithelial deposition of collagenous fibers, mediated by transforming growth factor (TGF)-beta secretion from mucosal eosinophils that stimulates fibroblasts to deposit extracellular matrix $[9,10]$. This ongoing remodeling process can lead to reduction of esophageal caliber, stricture formation, and subsequent food bolus impactions $[11,24]$. In addition, subepithelial fibrosis can lead to motility disturbances and thereby alter the esophageal clearance function and predispose to acid-related disorders [25]. A total of $83.3 \%$ of our patients had subepithelial fibrosis. This high proportion of patients with fibrosis is not surprising, bearing in mind a diagnostic delay of typically several years before diagnosis of EoE and that untreated inflammation leads to subepithelial fibrosis over time [11,26]. Furthermore, our results are in accordance with findings from an international EoE research group who documented subepithelial fibrosis in $>90 \%$ of their patients [19]. Fibrosis in EoE seems to be at least partially reversible, in adult as well as pediatric patients, by the long-term use of oral topical steroids but also of elimination diets [27-30]. As such, the histologic assessment of subepithelial fibrosis allows clinicians to understand the severity of esophageal remodeling.

A second advantage of sampling subepithelial tissue is that it can help pathology technicians to correctly orient the biopsy specimens before fixing them in formalin. The correct basoapical biopsy orientation (epithelial side up) provides sections that allow the pathologist to assess all EoE-associated features [21]. As such, correct orientation biopsy of the biopsy sample serves as a quality criterion in histopathology.

We found that both the static jaw forceps models (Olympus FB$11 \mathrm{~K}-1$ and Olympus FB-45Q-1) allowed sampling of subepithelial tissue in $>90 \%$ of biopsies and were therefore superior to the alligator jaw forceps and the large-capacity forceps. Furthermore, the static jaw forceps models sampled a significantly larger area of subepithelial tissue compared with the alligator jaw and the large-capacity forceps models. The highest rate of poorly oriented biopsies $(27.5 \%)$ was found for the large-capacity forceps which was also the model that provided the lowest frequency of subepithelial tissue. We conclude that static jaw forceps models are superior to alligator or large-capacity forceps in sampling a larger amount of subepithelial tissue.

Our study has several strengths and also some weaknesses. We present the first study to systematically assess the performance of four different biopsy forceps models in providing subepithelial tissue. All the biopsies were performed by a single EoE expert following a standardized protocol. All the EoE samples were processed at a single expert pathology laboratory and all samples were reviewed by a single EoE expert pathologist. As a first limitation, it is important to point out that the goal of the study was to evaluate the performance of different biopsy forceps models in providing subepithelial tissue, and not to assess the clinical relevance of sampling subepithelial tissue. As such, the clinical impact of sampling subepithelial tissue in adult EoE patients still needs to be evaluated. Second, our study methodology relies on the observation that the frequency and severity of subepithelial fibrosis do not differ between the proximal and distal esophagus in adult EoE patients. No comments can currently be made regarding fibrosis distribution in pediatric EoE patients. Third, the sample size of 240 esophageal biopsies in 30 patients is too low to draw definitive conclusions regarding the safety of subepithelial tissue sampling. Finally, we did not perform an in-depth cost-effectiveness analysis when comparing the single-use forceps versus multi-use forceps models. Considering that multi-use forceps models (each costing about 400 euros) are typically used in 15-20 patients before being discarded, the costs per-patient are comparable to that of a single-use forceps (cost about 20 euros).

In conclusion, we compared biopsy sampling of subepithelial tissue using four different forceps models, and found that use of a static jaw forceps allows the endoscopist to sample subepithelial tissue in more than $90 \%$ of all biopsies, and provides a greater amount of subepithelial tissue, when compared with alligator and large-capacity forceps. Obtaining biopsies using forceps models that are more likely to sample subepithelial tissue appears to be safe. Obtaining deeper biopsies will increase our understanding of the pathogenesis of EoE. The clinical relevance of assessing subepithelial tissue in adult EoE patients still needs to be evaluated.

Competing interests: Christian Bussmann, Nadine Haas, Sébastien Godat, Christine Sempoux, and Hans-Uwe Simon have no relevant financial, professional, or personal relationships to disclose. Alain M. Schoepfer has received consulting fees and/or speaker fees and/or research grants from: AstraZeneca, Switzerland; Aptalis Pharma; Dr. Falk Pharma, Germany; Glaxo Smith Kline; Nestlé, Switzerland; and Novartis, Switzerland. Ekaterina Safroneeva has received consulting fees from Aptalis Pharma, and from Novartis, Switzerland. Alex Straumann has received consulting fees and/or speaker fees and/or research grants from: Actelion, Switzerland; AstraZeneca, Switzerland; Aptalis Pharma; Dr. Falk Pharma, Germany; Glaxo Smith Kline; Nestlé, Switzerland; Novartis, Switzerland; Pfizer; and Regeneron Pharmaceuticals.

\author{
Institutions \\ Institute of Pathology, Viollier AG, Basel, Switzerland \\ 2 Division of Gastroenterology and Hepatology, Centre Hospitalier Universitaire \\ Vaudois (CHUV), Lausanne, Switzerland \\ ${ }^{3}$ Institute of Social and Preventive Medicine, University of Bern, Switzerland \\ ${ }^{4}$ Division of Pathology, Centre Hospitalier Universitaire Vaudois (CHUV), \\ Lausanne, Switzerland \\ ${ }^{5}$ Institute of Pharmacology, University of Bern, Bern, Switzerland \\ ${ }^{6}$ Swiss EoE Clinic, Praxis Römerhof, Olten, Switzerland
}

\section{Acknowledgments}

$\nabla$

The work was supported by grants from the Swiss National Science Foundation (32003B_135665 to A.M.S. and A.S. and 32003B_160115/1 to A.M.S.).

\section{References}

1 Liacouras CA, Furuta GT, Hirano I et al. Eosinophilic esophagitis: updated consensus recommendations for children and adults. J Allergy Clin Immunol 2011; 128: 3-20

2 Furuta GT, Katzka DA. Eosinophilic esophagitis. N Engl J Med 2015; 373: $1640-1648$

3 Giriens B, Yan P, Safroneeva E et al. Escalating incidence of eosinophilic esophagitis in Canton of Vaud, Switzerland, 1993-2013: a populationbased study. Allergy 2015; 70: 1633-1639

4 Riou PJ, Nicholson AG, Pastorino U. Esophageal rupture in a patient with idiopathic eosinophilic esophagitis. Ann Thorac Surg 1996; 62: 1854 1856

5 Saffari H, Peterson KA, Fang JC et al. Patchy eosinophil distributions in an esophagectomy specimen from a patient with eosinophilic esopha- 
gitis: implications for endoscopic biopsy. J Allergy Clin Immunol 2012; 130: $798-800$

6 Fox VL, Nurko S, Teitelbaum JE et al. High-resolution EUS in children with eosinophilic "allergic" esophagitis. Gastrointest Endosc 2003; 57: $30-36$

7 Straumann A, Conus S, Degen L et al. Long-term budesonide maintenance treatment is partially effective for patients with eosinophilic esophagitis. Clin Gastroenterol Hepatol 2011; 9: 400-409

8 Fontillon M, Lucendo AJ. Transmural eosinophilic infiltration and fibrosis in a patient with non-traumatic Boerhaave's syndrome due to eosinophilic esophagitis. Am J Gastroenterol 2012; 107: 1762

9 Aceves SS, Newbury RO, Dohil $R$ et al. Esophageal remodeling in pediatric eosinophilic esophagitis. J Allergy Clin Immunol 2007; 119: $206-$ 212

10 Mishra A, Wang M, Pemmaraju VR et al. Esophageal remodeling develops as a consequence of tissue specific IL-5-induced eosinophilia. Gastroenterology 2008; 134: 204-214

11 Schoepfer AM, Safroneeva E, Bussmann C et al. Delay in diagnosis of eosinophilic esophagitis increases risk for stricture formation, in a timedependent manner. Gastroenterology 2013; 145: 1230-1236

12 Kagalwalla AF, Akhtar N, Woodruff SA et al. Eosinophilic esophagitis: epithelial mesenchymal transition contributes to esophageal remodeling and reverses with treatment. J Allergy Clin Immunol 2012; 129: $1387-1396$

13 Safroneeva E, Straumann A, Coslovsky $M$ et al. Symptoms have modest accuracy in detecting endoscopic and histologic remission in adults with eosinophilic esophagitis. Gastroenterology 2016; 150: 581 -590

14 Sharaf RN, Shergill AK. ASGE Standards of Practice Committee. et al. Endoscopic mucosal tissue sampling. Gastrointest Endosc 2013; 78: $216-224$

15 Schoepfer AM, Panczak R, Zwahlen M et al. How do gastroenterologists assess overall activity of eosinophilic esophagitis in adult patients? Am J Gastroenterol 2015; 110: 402 - 414

16 Bussmann C. Requirements of the pathologist to the endoscopist (biopsy sampling). Dig Dis 2014; 32: 74-77

17 Dellon ES, Gonsalves N, Hirano I et al. ACG clinical guideline: evidence based approach to the diagnosis and management of esophageal eosinophilia and eosinophilic esophagitis. Am J Gastroenterol 2013; 108: 679-692

18 Molina-Infante J, Bredenoord AJ, Cheng $E$ et al. Proton pump inhibitorresponsive oesophageal eosinophilia: an entity challenging current diagnostic criteria for eosinophilic oesophagitis. Gut 2016; 65: 524-531
19 Schoepfer AM, Panczak R, Zwahlen M et al. How do gastroenterologists assess overall activity of eosinophilic esophagitis in adult patients? Am J Gastroenterol 2015; 110: 402-414

20 Hirano I, Moy N, Heckman MG et al. Endoscopic assessment of the oesophageal features of eosinophilic esophagitis: validation of a novel classification and grading system. Gut 2013; 62: 489-495

21 Bussmann C, Straumann A. Eosinophilic esophagitis: the diagnostic contribution of pathology. Pathologe 2013; 34: 110-117

22 Schoepfer AM, Hirano I, Katzka D. Eosinophilic esophagitis: overview of clinical management. Gastroenterol Clin North Am 2014; 43: 329344

23 Rieder F, Nonevski I, Ma J et al. T-helper 2cytokines, transforming growth factor $\beta 1$, and eosinophil products induce fibrogenesis and alter muscle motility in patients with eosinophilic esophagitis. Gastroenterology 2014; 146: 1266 - 1277

24 Straumann A, Bussmann C, Zuber M et al. Eosinophilic esophagitis: analysis of food impaction and perforation in 251 adolescent and adult patients. Clin Gastroenterol Hepatol 2008; 6: 598-600

25 Cheng E, Souza RF, Spechler SJ. Eosinophilic esophagitis: interactions with gastroesophageal reflux disease. Gastroenterol Clin North Am 2014; 43: $243-256$

26 Kuchen T, Straumann A, Safroneeva E et al. Swallowed topical corticosteroids reduce the risk for long-lasting bolus impactions in eosinophilic esophagitis. Allergy 2014; 69: $1248-1254$

27 Rajan J, Newbury RO, Anilkumar A et al. Long-term assessment of esophageal remodeling in patients with pediatric eosinophilic esophagitis treated with topical corticosteroids. J Allergy Clin Immunol 2016; 137: $147-156$

28 Lieberman JA, Morotti RA, Konstantinou GN et al. Dietary therapy can reverse esophageal subepithelial fibrosis in patients with eosinophilic esophagitis: a historical cohort. Allergy 2012; 67: 1299-1307

29 Lucendo AJ, Arias A, De Rezende LC et al. Subepithelial collagen deposition, profibrogenic cytokine gene expression, and changes after prolonged fluticasone propionate treatment in adult eosinophilic esophagitis: a prospective study. J Allergy Clin Immunol 2011; 128: 1037 1046

30 Abu-Sultaneh SM, Durst P, Maynard V et al. Fluticasone and food allergen elimination reverse sub-epithelial fibrosis in children with eosinophilic esophagitis. Dig Dis Sci 2011; 56: 97-102 